\title{
Investigating charm and beauty production and elliptic flow with leptons in $\mathrm{Pb}-\mathrm{Pb}$ collisions with ALICE
}

Martin Völkı* for the ALICE Collaboration

Physikalisches Institut, Eberhard Karls Universität Tübingen, Tübingen

E-mail: martin. andreas.volklecern.ch

\begin{abstract}
Measurements of leptons from heavy-flavour hadron decays in nucleus-nucleus collisions can help provide an insight into the interaction of heavy quarks with the Quark-Gluon Plasma. Both electrons and muons can be used for this purpose. Measurements in $\mathrm{Pb}-\mathrm{Pb}$ collisions at $\sqrt{s_{\mathrm{NN}}}=5.02 \mathrm{TeV}$ with ALICE show that both beauty and charm quarks interact strongly with the deconfined medium, showing suppression with respect to proton-proton collisions at high momenta and significant elliptic flow. The measurements can constrain the theoretical models and shed light on the mass dependence of the interactions.
\end{abstract}

European Physical Society Conference on High Energy Physics - EPS-HEP2019 -

10-17 July, 2019

Ghent, Belgium

${ }^{*}$ Speaker. 


\section{Introduction}

Heavy quarks are effective probes of the deconfined state of hadronic matter, the quark-gluon plasma (QGP). Due to their large masses they are produced almost exclusively in the initial hard scatterings of the nucleus-nucleus collision and therefore experience the entire evolution of the system. The large mass also means that perturbative methods can be used to understand the initial production down to zero momentum. Finally, there is a clear correspondence of the heavy quark in the medium and the heavy valence quark in the final-state hadrons.

The interaction with the medium is usually modeled as scatterings of the heavy quarks off the medium constituents. Here, collisional and radiative processes can be considered. Their relative contribution to the interaction is expected to be dependent on both the momentum and mass of the quark, motivating measurements at low and high transverse momenta $\left(p_{\mathrm{T}}\right)$ and for both charm and beauty. Measurements in the charm sector suggest that the thermalisation time for charm may well be of the order of the system lifetime[1,2]. An obvious candidate for a non-thermalized probe would thus be beauty quarks. These are however more difficult to reconstruct from their purely hadronic decays.

A useful property of heavy-flavour hadrons is their fairly large branching ratios to final states containing leptons $(\approx 10 \%)$. These can be measured by using the excellent particle identification capabilities of the ALICE apparatus [3]. Two particularly useful observables for measurements are the nuclear modification factor $R_{\mathrm{AA}}$, which compares $\mathrm{Pb}-\mathrm{Pb}$ collisions to an equivalent number of independent pp collisions, and the elliptic flow coefficient $v_{2}$, which quantifies the azimutal anisotropy of the particle production.

\section{Lepton measurements with ALICE}

Measurements of leptons from heavy-flavour decays with ALICE broadly consist of first selecting a sample of (mostly) leptons via particle identification and then finding out the contribution of heavy flavours to this sample.

Muons are measured in the pseudorapidity range $-4<\eta<-2.5$ using the forward muon spectrometer. They are identified by using the front absorber, corresponding to about 10 interaction lengths $\left(\lambda_{\mathrm{I}}\right)$ and the muon filter which rejects the remaining hadronic contamination.

Electrons are measured at midrapidity, typically with $|\eta|<0.8$. The main detector for particle identification here is the Time Projection Chamber (TPC), which has a signal based on the specific energy loss of charged particles in the gas $\mathrm{d} E / \mathrm{d} x$. The selection for the electron analyses is typically about $-0.5<n_{\sigma, \mathrm{e}}<3$, where $n_{\sigma, \mathrm{e}}$ is the deviation of the detector signal from the expectation value for electrons in units of the detector resolution. In addition, information from the time-of-flight detector (TOF, at lower $p_{\mathrm{T}}$ ) and the electromagnetic calorimeter (EMCal, towards higher $p_{\mathrm{T}}$ ) is used.

The separation of the leptonic background will be discussed in the context of the individual analyses.

\section{3. $R_{\mathrm{AA}}$ of muons from heavy-flavour decays}

At low transverse momenta, the main non-heavy-flavour source of muons is expected to be the 

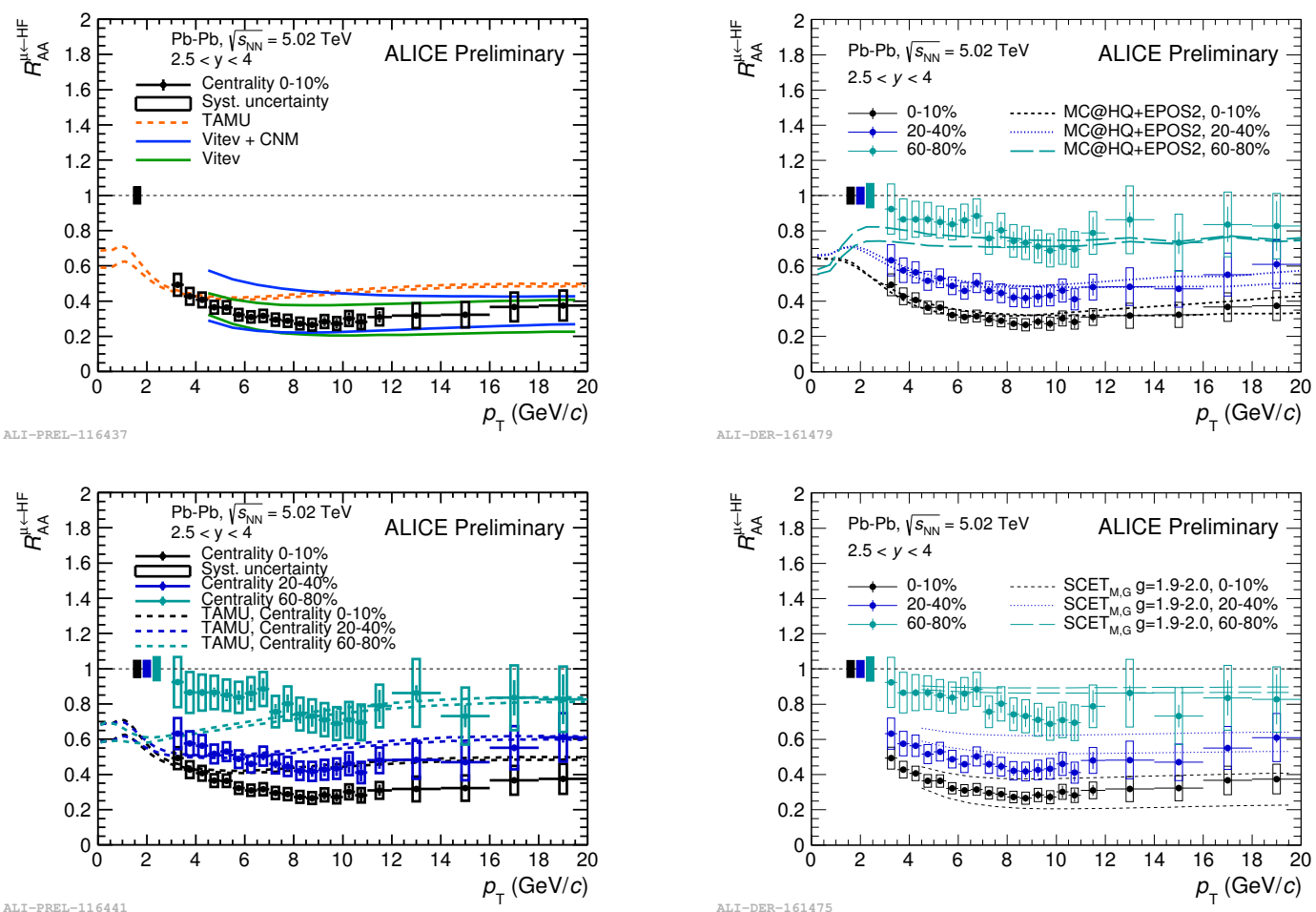

Figure 1: $R_{\mathrm{AA}}$ of heavy-flavour decay muons compared with models $[5,6,7]$.

decay of charged pions and kaons. This contribution is estimated from measurements performed at midrapidity. These are used as an input to tune detector simulations, which makes it possible to include effects of the decay geometry properly. Another contribution to the background are muons from the decay of charmonia, which were estimated based on measurements of muon pairs in the forward direction. At higher transverse momenta, $\mathrm{W}$ and $\mathrm{Z}$ boson decays become an important contribution. It is estimated from calculations of the POWHEG NLO event generator [4].

After subtracting the background and correcting for the detection and acceptance efficiencies, the yield can be compared to the pp case, leading to the nuclear modification factor shown in figure 1. The comparison to different models shows that these perform qualitatively well. Some do deviate from the measurement on a quantitative level, like the TAMU model [5] for peripheral collisions at low $p_{\mathrm{T}}$ and for central collisions at high $p_{\mathrm{T}}$.

\section{4. $R_{\mathrm{AA}}$ of electrons from heavy-flavour decays}

The dominant sources of electrons not from heavy-flavour decays are the decays of light mesons. This can either be a direct decay (e.g. $\pi^{0} \rightarrow e^{+} e^{-} \gamma$ ) or a decay to photons (e.g. $\pi^{0} \rightarrow \gamma \gamma$ ) which convert to an electron-positron pair in the detector material. The contribution can be estimated by making use of the small invariant mass of the pair. Figure 2 (left) shows the measured distribution of the electron-positron pair invariant mass compared to the scaled distribution for like-sign pairs. The excess from the low mass pairs is clearly visible and can then be used to estimate the dominant background electron contribution. 

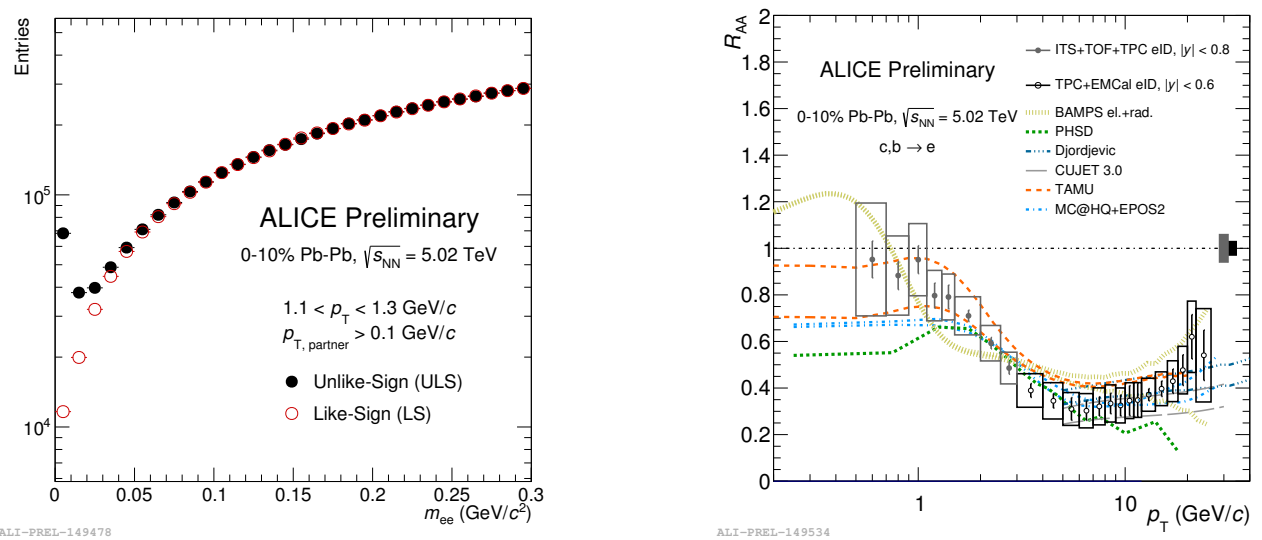

Figure 2: Left: Invariant mass distribution of reconstructed electron-positron pairs. Right: $R_{\mathrm{AA}}$ of heavyflavour decay electrons compared with models $[8,9,10,11,5,6]$.
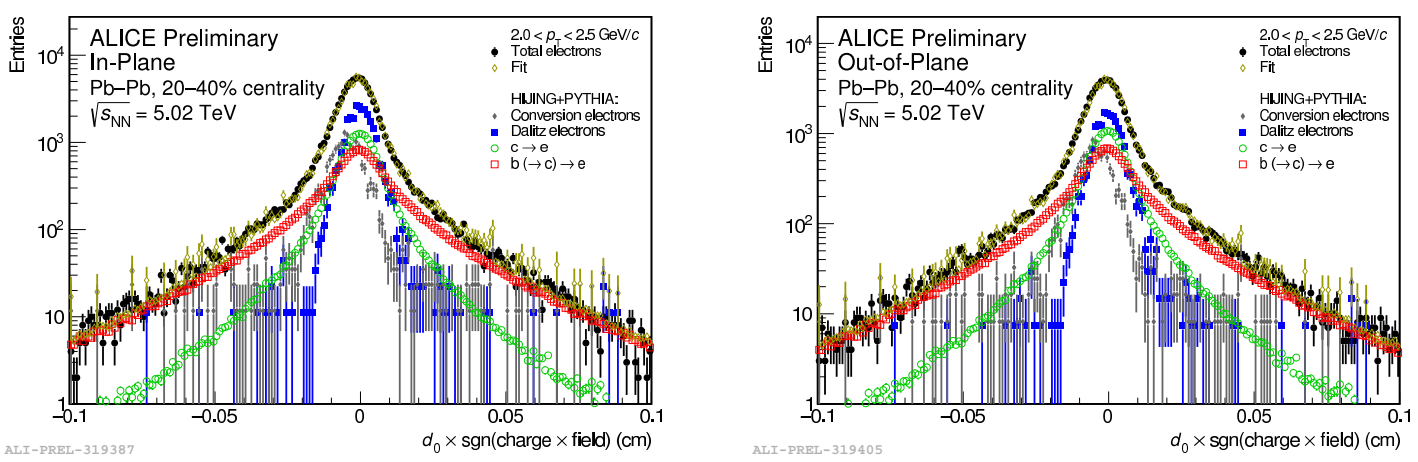

Figure 3: Examples of fits of the electron transverse impact parameter distributions in-plane (left) and outof-plane (right).

The resulting $R_{\mathrm{AA}}$ is shown on the right of figure 2. The model calculations are generally compatible with the measurement within the uncertainties.

\section{Beauty decay electron $R_{\mathrm{AA}}$}

Given the excellent measurements of the charm sector using hadronic decays, the information leptons give about beauty is particularly interesting. For this purpose, it is useful to separate the electron sources by their transverse impact parameter distributions. This motivates four categories: electrons from charm and beauty decays, electrons from photon conversions in the detector material and all others. The latter originate mainly from decays of light mesons close to the primary vertex. Thus, their distribution is mostly determined by the resolution of the tracks and the primary vertex. Electrons from photon conversions are produced in the direction of the photon, which is usually produced close to the primary vertex. Their distribution is thus also influenced by the bending of the tracks in the magnetic field and the distribution of the production vertices. The contributions are represented by templates of the distributions from detector simulations which are 

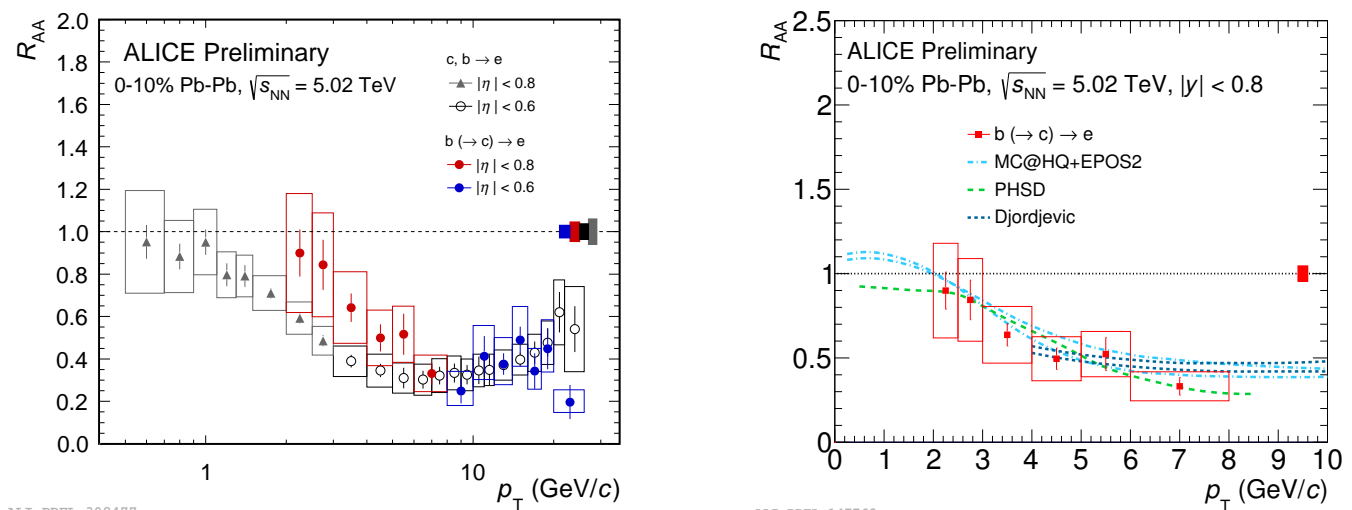

Figure 4: Measured $R_{\mathrm{AA}}$ of electrons from beauty-hadron decays. The left plot shows a comparison with the inclusive (charm and beauty) measurement, the right side shows a comparison with models $[6,9,10]$.
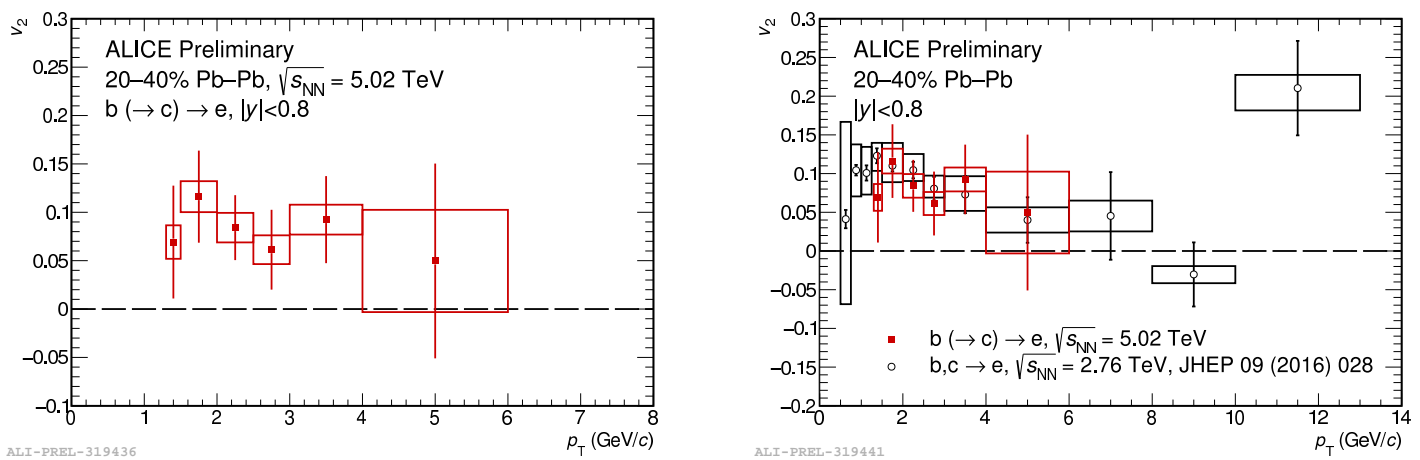

Figure 5: Measured $v_{2}$ of electrons from beauty-hadron decays. The right plot shows a comparison to the $v_{2}$ of electrons from beauty and charm at $\sqrt{s_{\mathrm{NN}}}=2.76 \mathrm{TeV}[13]$.

corrected for detector and physics effects. All contributions are extracted from the data by fitting the measured impact parameter distribution taking into account the statistical fluctuations in data and the templates [12]. Examples of such fits are shown in figure 3.

Figure 4 shows the resulting $R_{\mathrm{AA}}$ as a function of $p_{\mathrm{T}}$. At high $p_{\mathrm{T}}$, there is agreement with the inclusive case, which is expected as the relative contribution of beauty increases with $p_{\mathrm{T}}$ (left panel). The three models shown on the right panel give similar predictions and agree with the measurement within uncertainties.

\section{Beauty decay electron $v_{2}$}

The elliptic flow coefficient $v_{2}$ of beauty decay electrons can be extracted by comparing the yields within $45^{\circ}$ of the reconstructed event plane (figure 3, left) to those outside (figure 3, right). The result is shown in figure 5 .

The measured $v_{2}$ has central values around 0.1 . Assuming fully correlated systematic uncertainties, the significance of the deviation above zero in the first five bins is $\sim 3.5 \sigma$. The maximum is lower than for the D mesons, which reach values around $0.18[1,2]$. However, this difference is 
difficult to interpret directly due to the difference in kinematics between measuring the hadrons and measuring their decay daughters. Similar difficulties arise in comparing to the heavy-flavour decay electron results [13] shown on the right side of figure 5. A more thorough interpretation requires comparison to model calculations.

\section{Summary}

Together with the measurements of charm hadrons, measurements of leptons from heavyflavour hadron decays serve to provide a complete picture of the interaction of charm and beauty quarks with the deconfined medium. ALICE has performed measurements of the two basic quantities $R_{\mathrm{AA}}$ and $v_{2}$ for charm and beauty, showing that both interact significantly with the medium.

\section{References}

[1] ALICE Collaboration, $D$-meson azimuthal anisotropy in midcentral $\mathrm{Pb}$-Pb collisions at $\sqrt{\mathbf{S}_{\mathrm{NN}}}=\mathbf{5 . 0 2}$ TeV, Phys. Rev. Lett. 120 (2018) 102301 [1707.01005].

[2] ALICE Collaboration, Measurement of $D^{0}, D^{+}, D^{*+}$ and $D_{s}^{+}$production in $P b$ - $P b$ collisions at $\sqrt{\mathrm{s}_{\mathrm{NN}}}=5.02 \mathrm{TeV}$, JHEP 10 (2018) 174 [1804.09083].

[3] ALICE Collaboration, Performance of the ALICE Experiment at the CERN LHC, Int. J. Mod. Phys. A29 (2014) 1430044 [1 402.4476 ].

[4] S. Alioli, P. Nason, C. Oleari and E. Re, NLO vector-boson production matched with shower in POWHEG, JHEP 07 (2008) 060 [0805 . 4802].

[5] M. He, R. J. Fries and R. Rapp, Heavy Flavor at the Large Hadron Collider in a Strong Coupling Approach, Phys. Lett. B735 (2014) 445 [1401.3817].

[6] M. Nahrgang, J. Aichelin, P. B. Gossiaux and K. Werner, Influence of hadronic bound states above $T_{c}$ on heavy-quark observables in $\mathrm{Pb}+\mathrm{Pb}$ collisions at at the CERN Large Hadron Collider, Phys. Rev. C89 (2014) 014905 [1305.6544].

[7] Z.-B. Kang, F. Ringer and I. Vitev, Effective field theory approach to open heavy flavor production in heavy-ion collisions, JHEP 03 (2017) 146 [1610 . 02043$].$

[8] J. Uphoff, F. Senzel, O. Fochler, C. Wesp, Z. Xu and C. Greiner, Elliptic flow and nuclear modification factor in ultrarelativistic heavy-ion collisions within a partonic transport model, Phys. Rev. Lett. 114 (2015) 112301 [1401.1364].

[9] T. Song, H. Berrehrah, D. Cabrera, W. Cassing and E. Bratkovskaya, Charm production in $\mathrm{Pb}+\mathrm{Pb}$ collisions at energies available at the CERN Large Hadron Collider, Phys. Rev. C93 (2016) 034906 [1512.00891].

[10] M. Djordjevic, Heavy flavor puzzle at LHC: a serendipitous interplay of jet suppression and fragmentation, Phys. Rev. Lett. 112 (2014) 042302 [1307.4702].

[11] J. Xu, J. Liao and M. Gyulassy, Bridging Soft-Hard Transport Properties of Quark-Gluon Plasmas with CUJET3.0, JHEP 02 (2016) 169 [1508.00552].

[12] R. Barlow and C. Beeston, Fitting using finite monte carlo samples, Comp. Phys. Comm. 77 (1993) 219.

[13] ALICE Collaboration, Elliptic flow of electrons from heavy-flavour hadron decays at mid-rapidity in Pb-Pb collisions at $\sqrt{\mathrm{S}_{\mathrm{NN}}}=2.76 \mathrm{TeV}, \mathrm{JHEP} 09$ (2016) 028 [1606.00321]. 